\title{
OPEN Targeting CD38 in acute myeloid leukemia interferes with leukemia trafficking and induces phagocytosis
}

\author{
Meike Farber ${ }^{1}$, Yiyang Chen ${ }^{1,2}$, Lucas Arnold ${ }^{1}$, Michael Möllmann ${ }^{1}$, Eva Boog-Whiteside ${ }^{1}$, \\ Yu-An Lin ${ }^{1}$, H. Christian Reinhardt ${ }^{1}$, Ulrich Dührsen ${ }^{1}$ \& Maher Hanoun ${ }^{1} \bowtie$
}

Targeting the interaction between leukemic cells and the microenvironment is an appealing approach to enhance the therapeutic efficacy in acute myeloid leukemia (AML). AML infiltration induces a significant release of inflammatory cytokines in the human bone marrow niche which accelerates leukemogenesis. As the transmembrane glycoprotein CD38 has been shown to regulate cytokine release, we assessed the anti-leukemic potential of CD38 inhibition in AML. CD38 expression in AML cells proved to depend on microenvironmental cues and could be significantly enforced through addition of tretinoin. In fact, the anti-CD38 antibody daratumumab showed significant cytostatic efficacy in a 3D in vitro triple-culture model of AML, but with modest cell-autonomous cytotoxic activity and independent of $C D 38$ expression level. In line with a predominantly microenvironmentmediated activity of daratumumab in AML, CD38 inhibition significantly induced antibody-dependent phagocytosis and showed interference with AML cell trafficking in vivo in a xenograft transplantation model, but overall lacked robust anti-leukemic effects.

Acute myeloid leukemia (AML) is the most common acute leukemia in adults with increasing incidence with rising age ${ }^{1}$. Already at low disease burden, AML is marked by severe hematopoietic failure, which is the major cause of death. Additionally, despite intensive chemotherapeutic protocols, high relapse rates and primary resistance frequently occur, requiring further treatment intensification, including allogeneic hematopoietic stem cell transplantation ${ }^{1}$. However, many elderly AML patients are not eligible for intensive treatment protocols and therefore show disappointingly low overall survival rates with few patients surviving beyond five years of follow$u^{2}$. A major obstacle in AML therapy is persistence of quiescent leukemia-initiating cells, which are protected in their bone marrow niche ${ }^{3}$. Therefore, uncovering the microenvironmental regulation of AML cells and defining, ideally less toxic, niche-targeted therapies is of utmost need to make leukemia cells more vulnerable to genotoxic drugs and efficiently eradicate residual leukemia cells. Recently, we have gained increasing insight how AML cells are regulated in the bone marrow niche in murine models and humans ${ }^{4-6}$. AML infiltration induced a significant release of inflammatory cytokines in the human bone marrow, which proved to accelerate leukemogenesis ${ }^{7}$. The transmembrane glycoprotein CD38 appears to regulate cytokine release, adhesion, and cellular migration toward sites of inflammation ${ }^{8}$. CD38 is widely expressed on immune cells and its expression level depends on the maturation and activation state ${ }^{9}$. Besides, most AML blasts show high CD38 expression without obvious correlation with cytomorphological and genetic characteristics ${ }^{10}$. Indeed, CD38 monoclonal antibodies have shown high efficacies in multiple myeloma at different lines of therapy, which is exerted through both antibodymediated cytotoxicity, as well as modulation of immune surveillance in the niche ${ }^{11,12}$. Notably, the first-in-class human IgG1K anti-CD38 antibody daratumumab showed, throughout a number of clinical studies, a beneficial spectrum of side effects. Therefore, inhibition of CD38 in AML appears to be an appealing concept. Here, we show that CD38 inhibition leads to significant cytostatic efficacy in a 3D in vitro triple-culture model of AML, with modest cell autonomous cytotoxic activity. Daratumumab significantly induces antibody dependent phagocytosis in AML and shows interference with AML cell trafficking in vivo in a xenograft transplantation model.

${ }^{1}$ Department of Hematology and Stem Cell Transplantation, University Hospital Essen, Hufelandstraße 55, 45122 Essen, Germany. ${ }^{2}$ Division of Hematology and Oncology, Department of Medicine, Chang Gung Memorial Hospital, Chiayi, Taiwan. ${ }^{\boxplus}$ email: maher.hanoun@uk-essen.de 


\section{Results \\ CD38 inhibition results in high anti-leukemic efficacy in vitro independent of CD38 expression} level. To test if CD38 inhibition affects leukemia growth in AML, we applied the monoclonal antibody daratumumab on a series of different AML cell lines in mono-culture, as well as in co-culture to elaborate putative niche-mediated effects of daratumumab. AML cell lines were cultured with endothelial cells (human umbilical vein endothelial cells, HUVEC) or MS-5 fibroblasts, which have been shown before to significantly support AML cell line growth ${ }^{7}$. We could confirm that co-culture with stroma cells led to an increased expansion of AML cells (Fig. 1a). Daratumumab showed robust, but only minor cytotoxic effects on mono-cultured AML cells, which could not be significantly enhanced with increasing dosages of up to $10 \mu \mathrm{g} / \mathrm{ml}$ (Fig. 1a, Supplementary Fig. S1a). In contrast, in co-cultured AML cells daratumumab displayed increased anti-leukemic activity already at $0.1 \mu \mathrm{g} / \mathrm{ml}$, in particular for OCI-AML3, Kasumi-1 and MOLM-13 cells. Only minor anti-leukemic effects were observed in KG-1, OCI-M2, HL-60 and K562 cells. To test its clinical relevance, we treated a number of primary human AML or peripheral blood mononuclear cells from healthy donors with $0.1 \mu \mathrm{g} / \mathrm{ml}$ daratumumab. To better mimic the influence of the microenvironment on primary human AML cells, we co-cultured hematopoietic cells with either HUVEC or mesenchymal stem and progenitor cells grown as non-adherent spheres, hereafter referred to as mesenspheres, or both under serum-free conditions. Indeed, we observed that co-culture with either stroma cell type promoted growth of primary hematopoietic cells indicating that our in vitro system partially recapitulates features of the hematopoietic stem cell niche (Fig. 1b,c). Applying daratumumab on AML cells co-cultured with endothelial cells or mesenspheres resulted in significant reduction in AML cell numbers (Fig. 1b). The anti-leukemic effect was even more pronounced when culturing AML cells in 3D triple-culture with adherent endothelial cells and mesenspheres, where daratumumab induced reduction in AML cell number by $36 \%$. Again, no anti-leukemic effects were observed in mono-cultured AML cells. Of note, the anti-leukemic efficacy did not vary between AML samples of different genetic risk characteristics categorized according to the European LeukemiaNet recommendations ${ }^{1}$ (Supplementary Table 1). Further, we did not observe any substantial cytotoxic effects of daratumumab on healthy peripheral blood mononuclear cells (Fig. 1c) nor on stroma cells (Supplementary Fig. S1b). To exclude any unspecific effects of daratumumab, we applied next to media containing the carrier solution, $\operatorname{IgG}_{1}$ as well as $\mathrm{IgG}_{1}-\mathrm{b} 12$ monoclonal antibody as isotype controls and did not observe any cytotoxic effects in any control condition compared to daratumumab (Supplementary Fig. S1c). Further, similar cytostatic effects were observed when AML cells were treated with isatuximab, another antiCD38 antibody (Supplementary Fig. S1d,e).

Given the fact that daratumumab harbored AML-specific anti-leukemic activity, which was predominantly induced through microenvironment-mediated effects, we next asked whether CD38 expression on AML cells is influenced by microenvironmental cues and if the anti-leukemic activity of daratumumab depends on CD38 expression levels. Co-culture of AML cells with both HUVEC and mesenspheres led to a statistically significant three-fold increase of CD38 expression in AML cells, but no significant difference in healthy peripheral blood mononuclear cells (Fig. 2a). It has been shown that the CD38 gene contains a retinoic acid responsive element, which, upon treatment with ATRA, induces CD38 upregulation ${ }^{13}$. In fact, treatment with $0.1 \mu \mathrm{M}$ ATRA led to a 25-fold higher CD38 expression in AML cells independent of the culture condition, while healthy control cells only showed a three to sixfold increased expression (Fig. 2a). Adding ATRA to mono-, co- or triple-cultured primary human AML cells or mononuclear control cells did not lead to significantly increased anti-leukemic effects of daratumumab (Fig. 2b, Supplementary Fig. S2a). To further elaborate the dependency of the antileukemic activity of daratumumab on CD38 expression in AML, we correlated the CD38 expression of primary AML cells under different culture conditions with the degree of reduction of AML cell growth in vitro (Fig. 2c). We did not observe any consistent correlation of CD38 expression and anti-leukemic efficacy of daratumumab; only in primary AML cells co-cultured with HUVECs in the presence of ATRA the anti-leukemic activity of daratumumab depended on CD38 expression. Similarly, we did not observe robust correlation between CD38 expression and sensitivity to daratumumab in AML cell lines, mono- or co-cultured with HUVECs (Supplementary Fig. S2b). However, co-culture with MS-5 showed a significant correlation of CD38 expression and sensitivity to daratumumab treatment.

In summary, daratumumab showed robust, AML-specific anti-leukemic efficacy in vitro, which is at least partially mediated through microenvironmental cues and to a lower extent through direct cytotoxic effects. CD38 expression in AML cells is regulated through the microenvironment and can be significantly enforced through addition of ATRA, while the anti-leukemic efficacy of daratumumab does not stringently depend on the level of CD38 expression.

Daratumumab induces phagocytosis in AML. To further elucidate the underlying mechanisms of the anti-leukemic activity of daratumumab in AML, we assessed the effects of daratumumab on proliferation in the most drug-responsive AML cell lines. BrdU analysis did not show significant differences in proliferation upon treatment (Fig. 3a, Supplementary Fig. S3a). Neither did we observe an induction of apoptosis by annexin quantification by flow cytometry (Fig. 3b, Supplementary Fig. S3b) nor by measuring mRNA levels of apoptosisrelated genes (Supplementary Fig. S3c). Furthermore, daratumumab did not induce differentiation of AML cells as assessed by cytomorphology (data not shown). As daratumumab has been shown to induce multiple immunemediated cytotoxic effects in multiple myeloma ${ }^{12}$, we next aimed at assessing these mechanisms in AML. We evaluated daratumumab-induced complement-dependent cytotoxicity (CDC) in OCI-AML3 and Kasumi-1 cells as the most drug-responsive AML cell lines, which were preincubated with calcein-AM and treated with increasing concentrations of daratumumab or isotype control. Here, daratumumab did not induce significant cell death in the presence of normal human serum (10\%) (Fig. 3c and Supplementary Fig. S3d). Nor could we detect any capacity of daratumumab to induce antibody-dependent cellular cytotoxicity (ADCC) by Fc $\gamma$ R- 
A

OCI AML-3

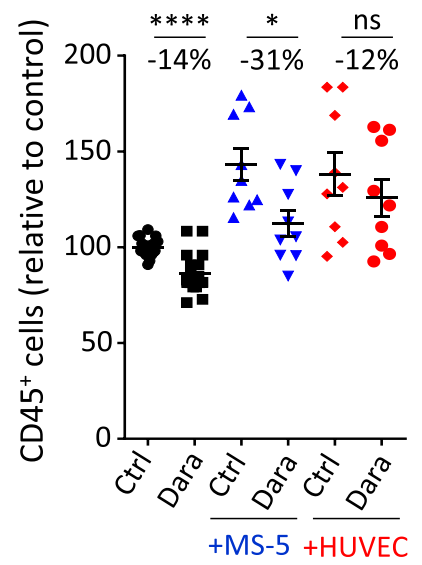

Kasumi-1

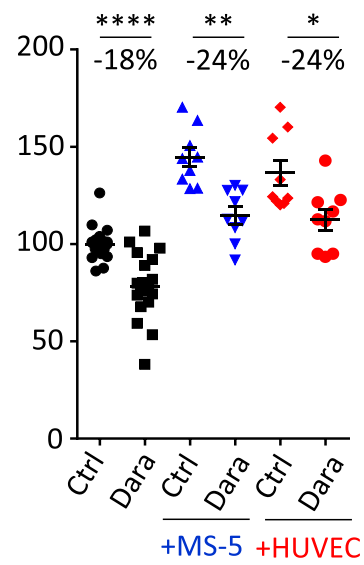

MOLM-13

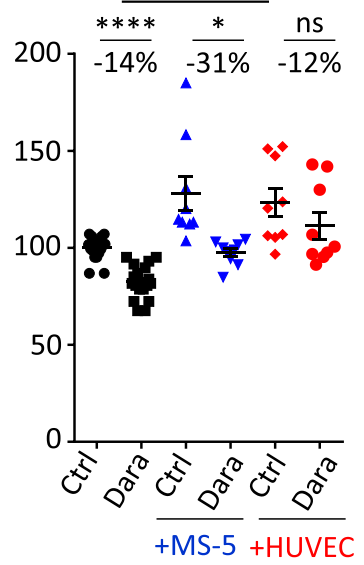

THP-1

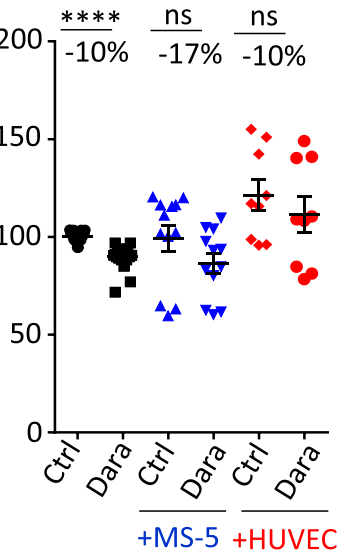

$\underline{\mathrm{KG}-1}$

$\underline{\mathrm{OCl}-\mathrm{M} 2}$

HL-60

K562
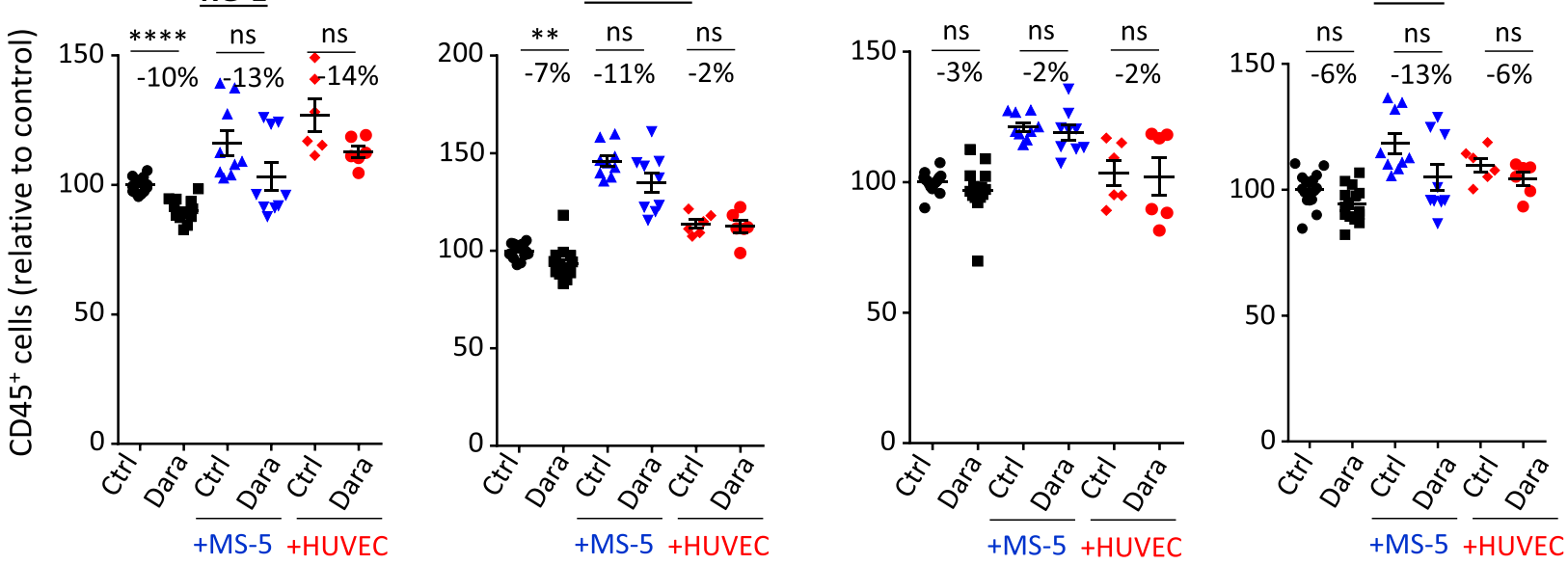

\section{B Primary AML cells}

\section{Healthy control cells}
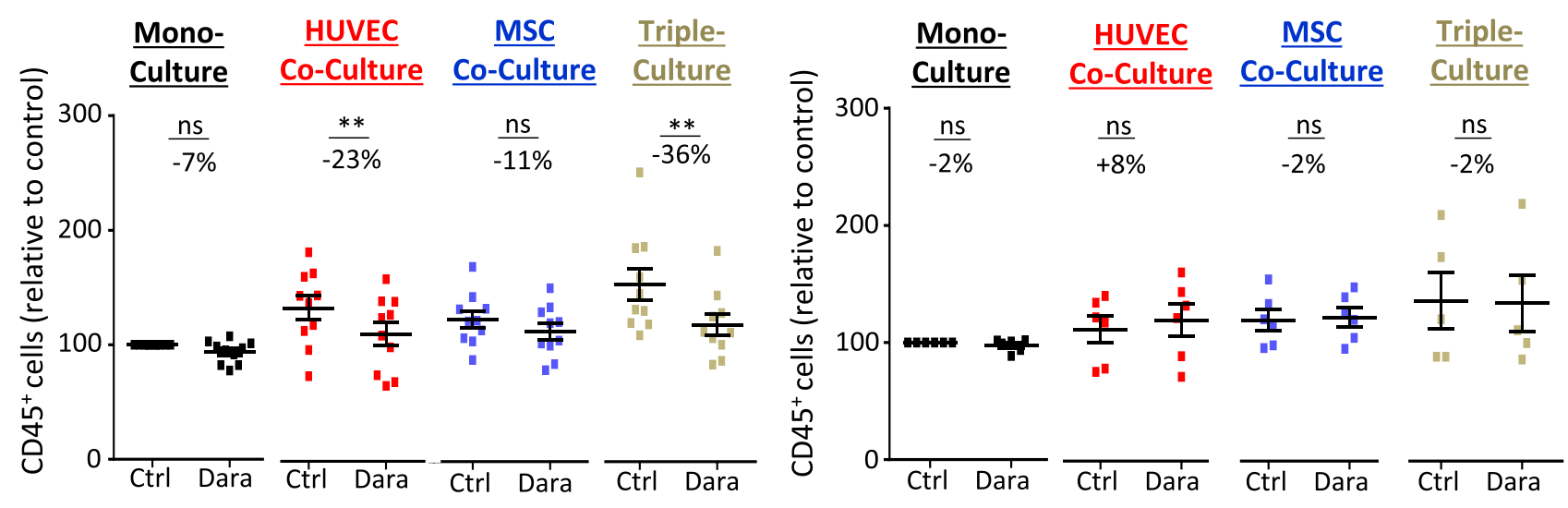

Figure 1. CD38 inhibition results in high anti-leukemic efficacy in vitro independent of CD38 expression level. (A) Human AML cell lines were either mono- or co-cultured with MS-5 stroma cells or HUVEC cells and treated with $0.1 \mu \mathrm{g} / \mathrm{ml}$ daratumumab or vehicle for 4 days. Absolute numbers of $\mathrm{CD}^{2} 5^{+}$cells were normalized to mono-cultured control, for each pair the mean change in cell count is given in percentage $(n=3)$. (B) Primary AML cells $(n=10)$ or $(C)$ healthy peripheral blood mononuclear cells $(n=6)$ were mono-, co- or triple-cultured with HUVEC and/or mesenspheres and treated with $0.1 \mu \mathrm{g} / \mathrm{ml}$ daratumumab or vehicle for 3 days. Absolute numbers of $\mathrm{CD}_{4} 5^{+}$cells were normalized to mono-cultured control. Each dot represents the mean of triplicates, for each pair the mean change in cell count is given in percentage. Data are shown as mean \pm SEM. n.s., not significant, ${ }^{*} p<0.05^{* *} p<0.01^{* * *} p<0.001{ }^{* * * *} p<0.0001$ as determined by unpaired student's t-test (A) and Wilcoxon signed-rank test (B,C). See also Supplementary Fig. S1. 
A
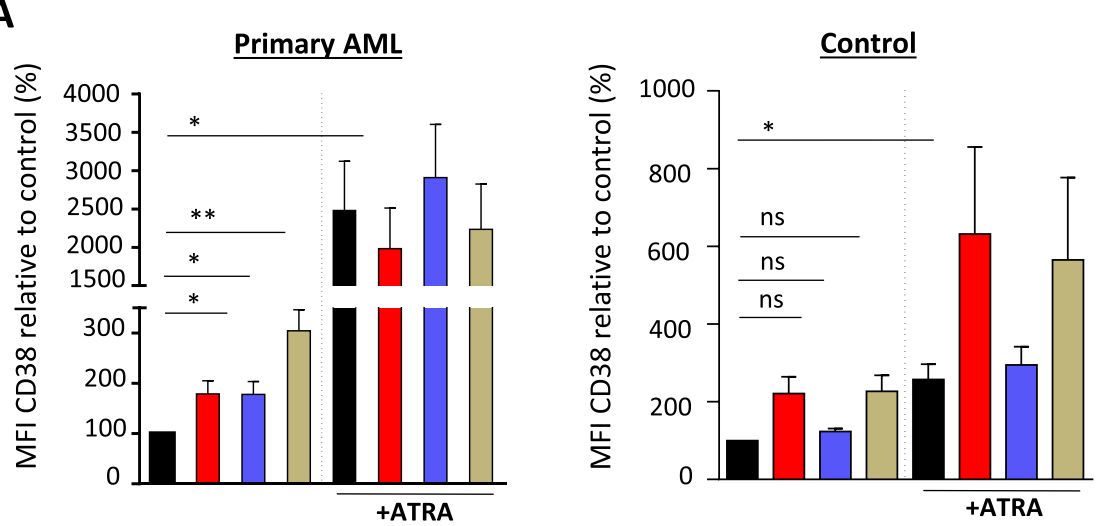

Mono-Culture

HUVEC Co-Culture

MSC Co-Culture

Triple-Culture

B
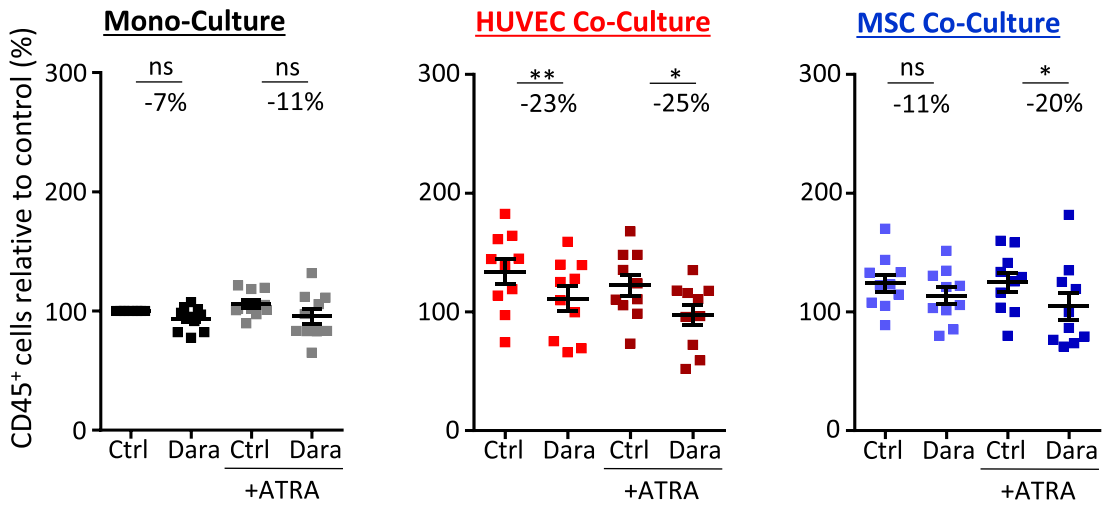

Triple-Culture

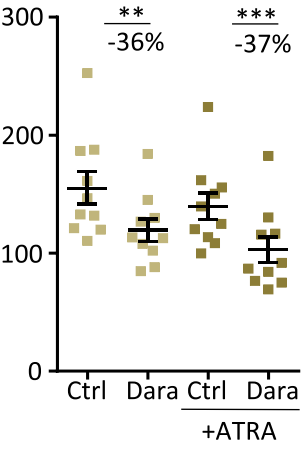

C
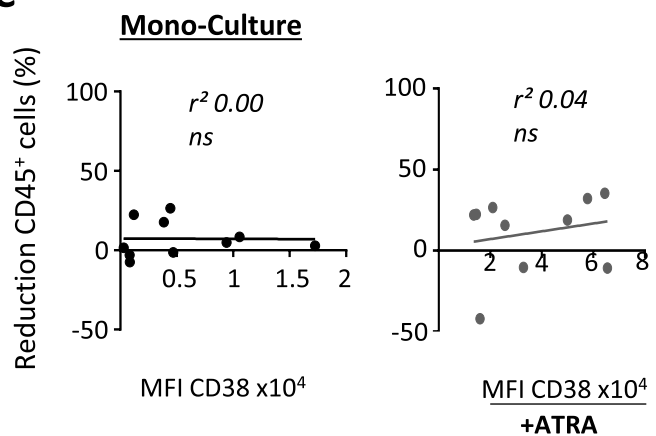

HUVEC-Co-Culture
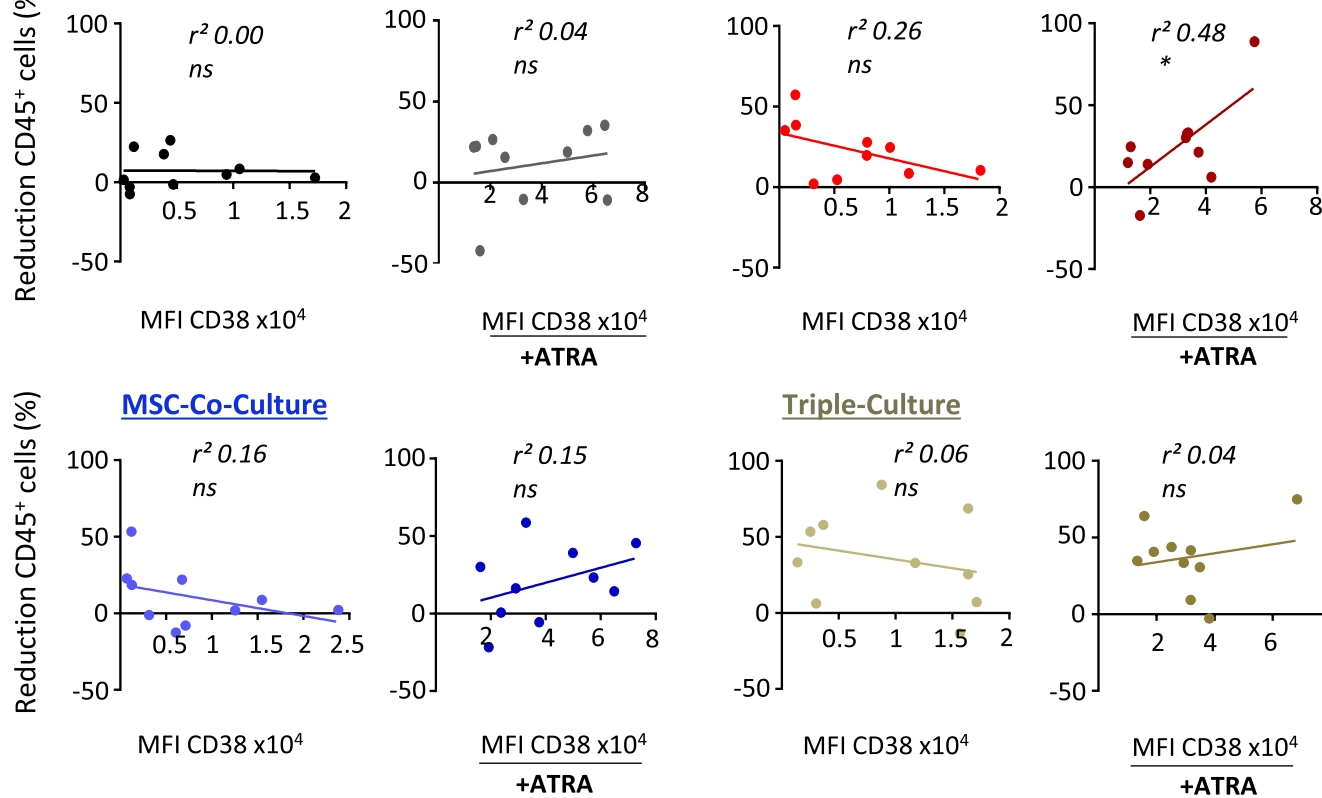

Triple-Culture
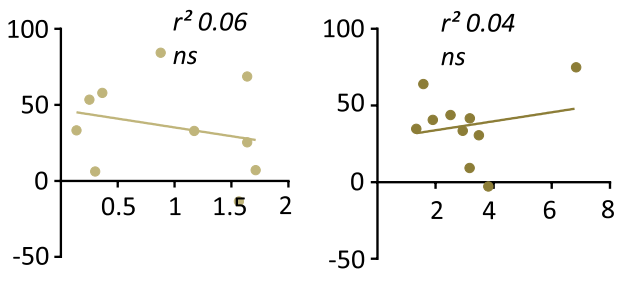

MFI CD38 $\times 10^{4}$

$\frac{\text { MFI CD38 } \times 10^{4}}{\text { +ATRA }}$ 
४Figure 2. CD38 expression of AML cells is regulated by microenvironmental cues and induced upon ATRA treatment. (A) Mean fluorescence intensity (MFI) of CD38 on primary AML cells $(n=10)$ and healthy peripheral blood mononuclear cells $(n=6)$ measured by flow cytometry, mono-, co- or triple-cultured with HUVEC and/or mesenspheres before and after treatment with $0.1 \mu \mathrm{M}$ all-trans-retinoic acid (ATRA), data normalized to mono-cultured control. (B) primary AML cells $(n=10)$ were mono-, co- or triple-cultured with HUVEC and/or mesenspheres. ATRA or vehicle was added at $0.1 \mu \mathrm{M}$ for 2 days followed by daratumumab treatment at $0.1 \mu \mathrm{g} / \mathrm{ml}$ or vehicle for another 3 days. Absolute numbers of CD $45^{+}$cells were normalized to mono-cultured control. Each dot represents the mean of triplicates, for each pair the mean change in cell count is given in percentage. (C) Correlation of anti-leukemic activity of daratumumab given as mean cell-count reduction with CD38 expression given as MFI measured by flow cytometry for each primary AML sample, mono-, co- or triple-cultured with HUVEC and/or mesenspheres $(n=10$ different AML donors, each primary sample was tested in triplicate). Data are shown as mean \pm SEM. n.s., not significant, ${ }^{\star} \mathrm{p}<0.05^{\star *} \mathrm{p}<0.01$ ${ }^{* * *} \mathrm{p}<0.001$ as determined by paired one-way ANOVA (A), Wilcoxon signed-rank test (B) and linear regression (C). See also Supplementary Fig. S2.

bearing effector cells at different drug concentrations in AML (Fig. 3d and Supplementary Fig. S3e). To evaluate the capacity to induce antibody-dependent cellular phagocytosis (ADCP) in AML, we generated macrophages from peripheral blood-derived monocytes stimulated with M-CSF and co-cultured with calcein-AM labeled AML cells in the presence of daratumumab or isotype control at the indicated concentrations. Daratumumab induced ADCP with increased phagocytosis of OCI-AML3 of around 1.64-fold (Fig. 3e) and for Kasumi-1 cells of 1.7-fold (Supplementary Fig. S3f.), which was not dose-dependent. To further substantiate these findings, we also applied primary human AML cells and documented for all five samples an increased phagocytosis of around 1.74-fold after treatment with daratumumab (Fig. 3f-g). In summary, in accordance to the minor cellautonomous anti-leukemic effects of daratumumab in AML, we did not observe any significant impact on AML proliferation, apoptosis, differentiation nor CDC and ADCC, while daratumumab strikingly induced phagocytosis of AML cells. As endothelial cells are known to harbor phagocytic capacities ${ }^{14}$, this might explain the significant microenvironment-mediated anti-leukemic effects of daratumumab in our 3D triple-culture model. Next, we evaluated whether daratumumab interferes in the interaction of mesenchymal stem and progenitor cells with AML cells. It has been previously shown that AML cells communicate with stroma cells through transfer of mitochondria ${ }^{15}$. In multiple myeloma mitochondrial transfer occurs through formation of nanotubules which was dependent on CD38 expression ${ }^{16}$. To test whether daratumumab disrupts the mitochondrial transfer and herewith the communication of AML cells with stroma cells, we traced mitochondria using MitoTracker ${ }^{\mathrm{Tm}}$ dye. In fact, we could prove that stroma cells transfer significant amounts of mitochondria to AML cells, while stroma cells only incorporate a limited amount of AML-derived mitochondria (Fig. 3h, Supplementary Fig. S4ab). Adding daratumumab to MOLM-13, OCI-AML-3 or Kasumi-1 cells co-cultured with HUVECs or MS-5 did not affect mitochondria transfer between AML and stroma cells (Fig. 3 h, Supplementary Fig. S4a-b). Due to possible dye efflux, we also quantified mtDNA in sorted AML cells after co-culture with HUVEC or MS-5 cells. After one day of co-culture there was a trend for decreased mitochondrial transfer upon daratumumab treatment, which disappeared after three days (Fig. 3i, Supplementary Fig. S4c).

As human CD38 was shown to bind to CD31, which is highly expressed on endothelial cells, we assumed a putative role in interference with adhesion and circulation of leukemia cells ${ }^{17}$. Therefore, we analyzed the gene expression profile of endothelial and hematopoietic cells purified by flow cytometry after triple-culture with either AML cells or control mononuclear cells. We assessed the expression of genes associated with migration and retention (CXCR4, CXCL12), adhesion (ICAM-1, VCAM-1, E-selectin, PECAM-1) and angiogenesisrelated factors (Angiopoietin-1, VEGF, VE-cadherin) in mono- or co-cultured cells treated with either ATRA, daratumumab or both. However, we did not observe any differences in mRNA levels neither in AML cells nor endothelial cells co-cultured with either AML or control peripheral blood mononuclear cells after treatment with daratumumab (Supplementary S5a-b). In conclusion, the microenvironment-mediated anti-leukemic activity of daratumumab is not due to interference with mitochondrial transfer nor in the expression of adhesion-, migration- and angiogenesis-related factors in vitro.

Daratumumab interferes with AML cell trafficking. To test the anti-leukemic activity of daratumumab in a clinically relevant in vivo model, we transplanted different primary human AML cells into immunodeficient NSG mice. As shown before, secondary transplantation of primary human AML cells allowed robust engraftment without any conditioning and, therefore, avoiding additional influence on the microenvironment ${ }^{7}$. Given the robust upregulation of CD38 expression in AML upon ATRA treatment (Fig. 2a), all mice were treated with ATRA in addition to daratumumab or $\operatorname{IgG}_{1}$ control (Fig. $4 \mathrm{a}$ ). With a mean leukemia burden of $\sim 7 \%$ $($ mean \pm 1$)$ in the peripheral blood, mice were randomized and weekly treated on 4 consecutive days with ATRA $(10 \mathrm{mg} / \mathrm{kg})$, as well as daratumumab $(8 \mathrm{mg} / \mathrm{kg})$ or $\mathrm{IgG}_{1}$ isotype control on the third day of each week for in total 4 weeks and analyzed 6 days after the end of treatment (Fig. $4 a$ ). Indeed, we could prove that addition of ATRA induced CD38 expression in AML cells in $\mathrm{IgG}_{1}$-treated control mice, although to a lower extend than in vitro (Supplementary Fig. S6a). In 3 out of 5 experiments we observed a striking decrease in human AML cells in peripheral blood by $-30 \%$, while overall no significant reduction in leukemia burden was observed in bone marrow and spleen (Fig. 4b,c, Supplementary Fig. S6b). In line with the apparent anti-leukemic activity observed in the peripheral blood, leukocyte counts were significantly lower in daratumumab-treated mice (Fig. 4d). After daratumumab treatment, bone marrow cellularity was slightly increased, while we did not observe obvious histological differences in bone marrow architecture, in particular no obvious differences in the amount of reticular 
Figure 3. Daratumumab induces phagocytosis in AML. (A) Cell proliferation analysis using BD BrdU flow assay. Left, representative flow cytometry plots, right quantification for OCI-AML-3 cells mono- or co-cultured with MS- 5 or HUVEC after treatment with $0.1 \mu \mathrm{g} / \mathrm{ml}$ daratumumab or IgG $_{1}$ control for 4 days, statistical analyses are given below ( $\mathrm{n}=3$ independent experiments). (B) Quantification of apoptotic cells by Annexin $\mathrm{V}$ assay of OCI-AML-3 cells mono- or co-cultured with MS-5 or HUVEC after treatment with $0.1 \mu \mathrm{g} / \mathrm{ml}$ daratumumab or $\mathrm{IgG}_{1}$ control for 4 days ( $\mathrm{n}=3$ independent experiments). (C) Quantification of complementdependent-cytotoxicity (CDC). Calcein stained OCI-AML-3 cells were incubated in medium supplemented

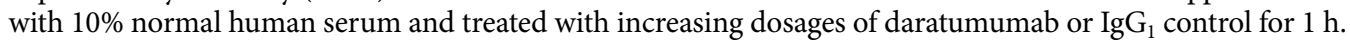
(D) Quantification of antibody-dependent cell-mediated cytotoxicity (ADCC). Calcein stained OCI-AML-3

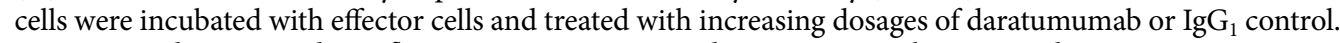
For CDC and ADCC, calcein-fluorescence was measured in supernatant by spectrophotometry. Lysis was calculated by using detergent as maximum and medium as minimum control ( $\mathrm{n}=3$ independent experiments). (E-G) Quantification of antibody-dependent phagocytosis (ADCP). Macrophages were cultured with calcein stained OCI-AML-3 ( $\mathrm{n}=3$ independent experiments) (E) as well as primary AML cells ( $\mathrm{n}=5$ different AML donors, each primary sample was tested in triplicate) (F) and treated with increasing dosages of daratumumab or $\mathrm{IgG}_{1}$ control. $\mathrm{CD}^{+} 6^{+}$calcein $^{+}$phagocyting macrophages were measured by flow-cytometry. Each dot represents the mean of triplicates. $(\mathbf{G})$ Representative flow cytometry plot of one calcein stained primary AML sample co-cultured with macrophages showing the fraction of AML-phagocyting macrophages. (H) Tracking mitochondrial transfer in co-culture was performed by staining mitochondria with MitoTracker ${ }^{\mathrm{rx}}$. Left, MFI of MitoTracker ${ }^{\text {Tw }}$ in MOLM-13 cells after co-culture with MitoTracker ${ }^{\mathrm{rax}}$ stained HUVEC and treatment with $0.1 \mu \mathrm{g} / \mathrm{ml}$ daratumumab or IgG 1 control for $24 \mathrm{~h}$ or mono-culture of unstained MOLM-13 as control. Right, MFI of MitoTracker ${ }^{\text {tix }}$ in HUVEC after co-culture with MitoTracker ${ }^{\text {Tix }}$ stained MOLM-13 cells and treating with $0.1 \mu \mathrm{g} / \mathrm{ml}$ daratumumab or $\operatorname{IgG}_{1}$ control for $24 \mathrm{~h}$ or mono-culture of unstained HUVEC as control. Below, representative histogram of MFI of MitoTracker ${ }^{\mathrm{Tm}}$, gated on MOLM-13 cells (left) or HUVEC (right) at the indicated culture conditions and treatment with $0.1 \mu \mathrm{g} / \mathrm{ml}$ daratumumab or IgG $_{1}$ control. (I) Indicated AML cells were co-cultured with either HUVEC (top) or MS- 5 cells (bottom) for 24 h, sorted by flow cytometry and mRNA expression levels were measured by rt-PCR for either human mitochondrial DNA ( $h m t D N A)$ after HUVEC co-culture or mouse mitochondrial DNA ( $m m t D N A)$ after MS-5 co-culture, relative to ncDNA. Data are shown as mean \pm SEM. n.s., not significant, ${ }^{\star} \mathrm{p}<0.05^{\star * * *} \mathrm{p}<0.0001$ as determined by unpaired student's t-test (A, B, E, H, Mann-Whitney-U Test (C, D, I), and Wilcoxon signed-rank test (F). See also Supplementary Fig. S3-5.

fibers (Fig. 4d, Supplementary Fig. S6c). Notably, within one experiment, we observed a stronger anti-leukemic activity of daratumumab, where the average leukemia burden in the control group was with 55\% AML infiltration comparably low. With the assumption that the anti-leukemic activity of daratumumab might be more pronounced at lower leukemia burden, we combined daratumumab treatment with a chemotherapeutic agent. For that, we added $20 \mathrm{mg} / \mathrm{kg}$ cytarabine on 4 consecutive days of the first week of treatment to the above-mentioned scheme (Fig. 4e). While the overall leukemia burden was comparably low, daratumumab treatment did not result in any significant differences in leukemia burden nor cellularity compared to $\operatorname{IgG}_{1}$ isotype treated controls (Fig. 4f, Supplementary Fig. S6d). Given the obvious redistrubition of leukemia cells after daratumumab treatment, we wondered if CD38 inhibition affects AML cell trafficking in vivo. For that, we treated NSG mice with daratumumab $(8 \mathrm{mg} / \mathrm{kg})$ or IgG $_{1}$ isotype control and $24 \mathrm{~h}$ later mice were transplanted with primary human AML cells. Sixteen hours after transplantation, we found three- to five-fold less AML cells in bone marrow and spleen, respectively, indicating an impaired homing of AML cells after daratumumab treatment (Fig. 4g). To elucidate the effects of daratumumab on AML/endothelial cell interactions, we assessed the transendothelial migration capacity of primary human AML cells. In fact, daratumumab significantly impaired transendothelial migration of AML cells by almost 50\%, while a direct cytotoxic effect after $16 \mathrm{~h}$ was ruled out (Fig. 4h, Supplementary Fig. S6e). Nevertheless, we cannot completely rule out that the lower number of AML cells in bone marrow and spleen after transplantation are due to immune-mediated cytotoxic effects of daratumumab. In summary, CD38 inhibition interferes in AML cell trafficking resulting in lower leukemia burden particularly in the peripheral blood, but overall does not show robust anti-leukemic activity as monotherapy or in combination with cytarabine in vivo.

\section{Discussion}

Disrupting the microenvironmental interplay with AML cells is an appealing approach to target leukemiainitiating cells and putatively increases their sensitivity to genotoxic influences. Both cell-autonomous and microenvironment-derived inflammatory signals promote clonal hematopoiesis within an aged bone marrow microenvironment and facilitate leukemogenesis, as for instance in B lineage malignancies as well as myeloid neoplasms ${ }^{18,19}$. Targeting inflammatory signals mitigates clonal hematopoiesis in TET2-mutated preleukemic stem and progenitor cells ${ }^{20}$, blocking IL- 6 signaling delays chronic myeloid leukemia development ${ }^{21}$ and interfering in the JAK/STAT pathway shows anti-leukemic activity in AML in vitro ${ }^{7}$, which prove the feasibility of targeting the inflammasome in myeloid diseases. Based on the prominent immunomodulatory function of CD38 and its wide expression in AML, we elaborated the effects of CD38 inhibition in AML. Daratumumab showed significant anti-leukemic activity in a 3D triple culture model, with only minor cell-autonomous efficacy, suggesting that daratumumab's anti-leukemic effects are predominantly mediated through the microenvironment. We could prove that CD38 expression in AML cells is regulated by niche cells and significantly increases after ATRA treatment. However, in contrast to a previous study, combination of daratumumab and ATRA did not increase anti-leukemic effects ${ }^{22}$. In line with the obvious microenvironment-mediated anti-leukemic activity, 
A

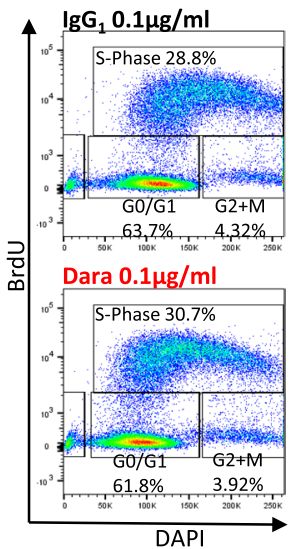

C

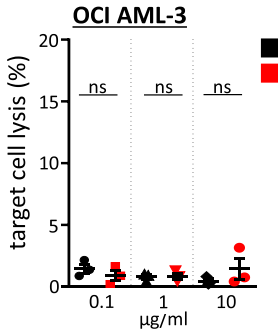

F

primary AML

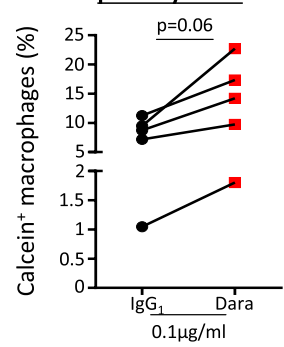

H

MOLM-13

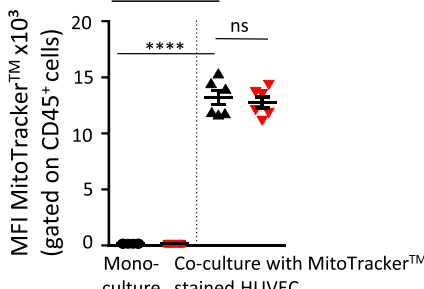
culture stained HUVEC

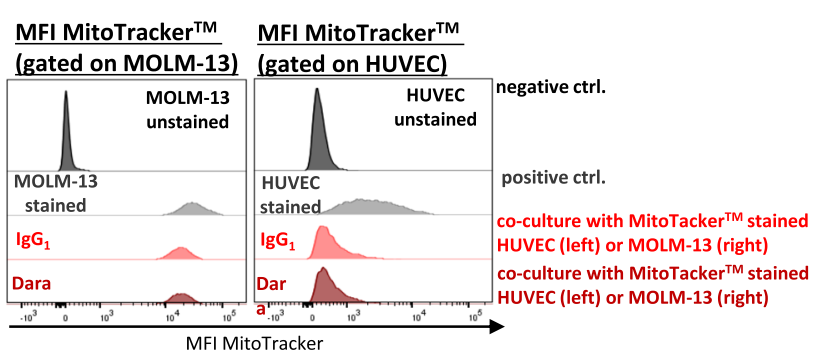

\begin{tabular}{|l|c|c|c|c|}
\hline OCl-AML3 & GO/G1 & \multicolumn{1}{|c|}{ G2/M } & \multicolumn{1}{l|}{ S } & apoptotic \\
\hline Mono-culture & ns & ns & ns & ns \\
\hline Co-culture MS-5 & ns & ns & ns & ns \\
\hline Co-culture HUVEC & ns & ns & ns & ns \\
\hline
\end{tabular}

D

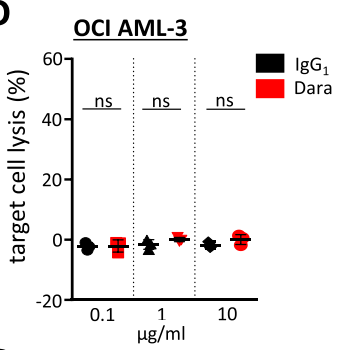

E
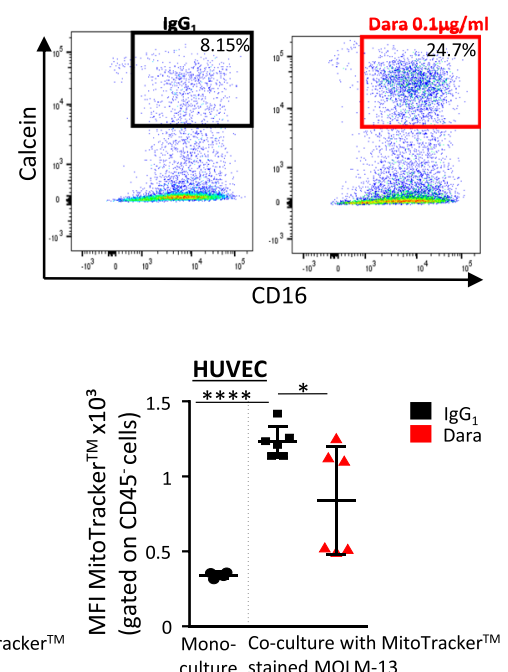
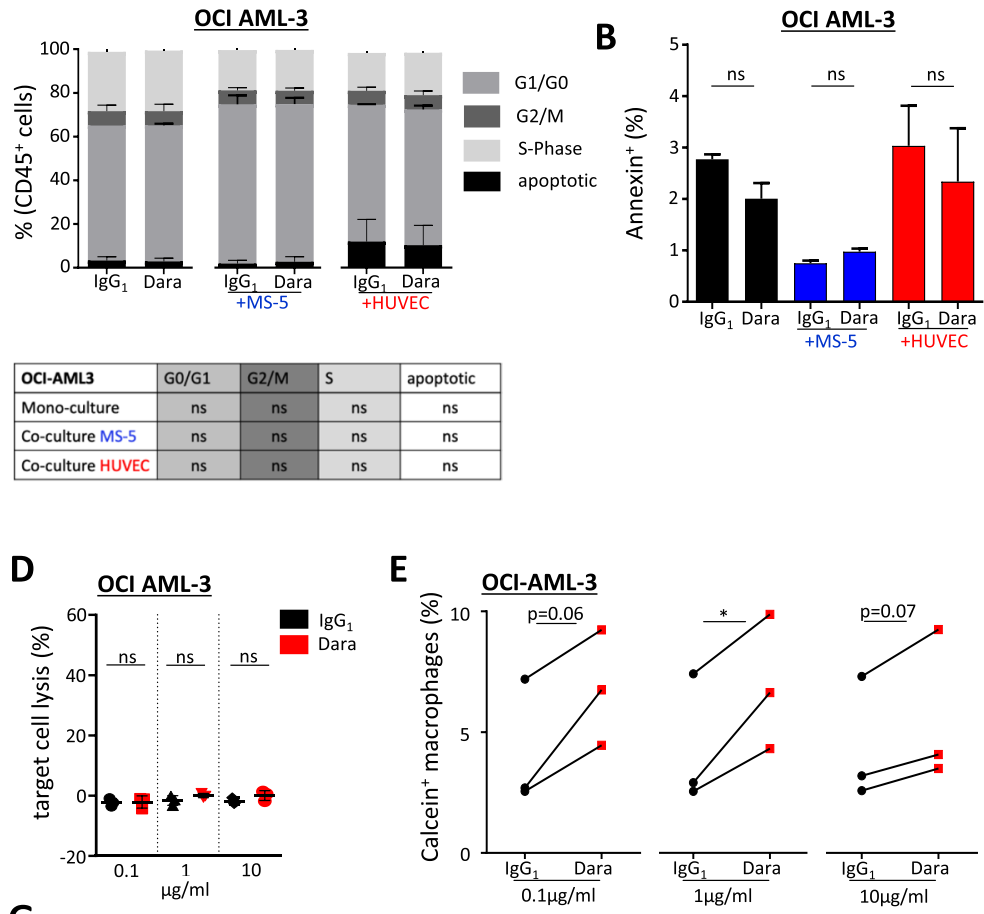

$\mathrm{p}=0.07$

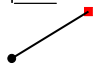

I
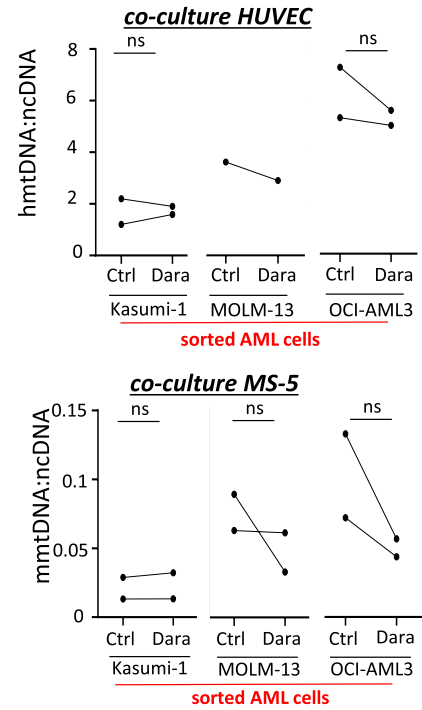
A

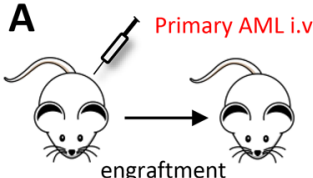

C
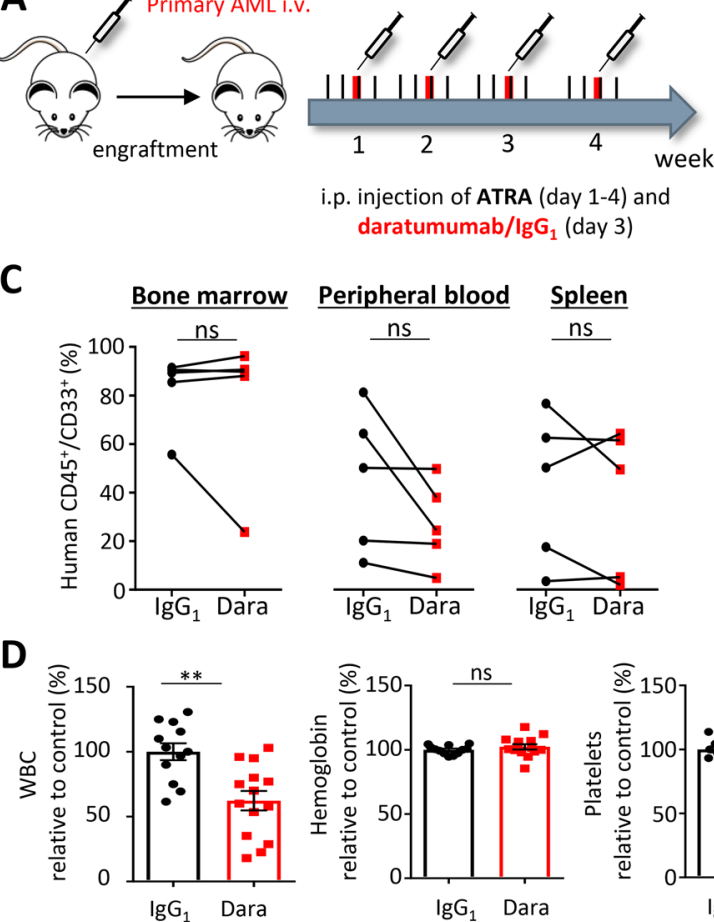

i.p. injection of ATRA (day 1-4) and

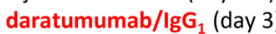

Peripheral blood

ns
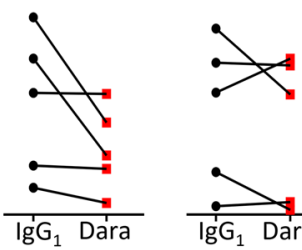

B

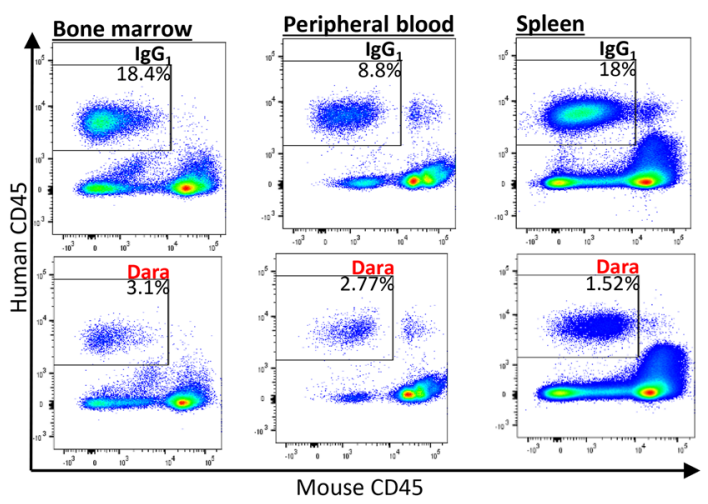

E

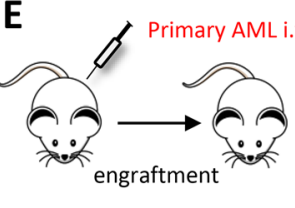

i.p. injection of cytarabine (day 1-4 in week 1)

i.p. injection of ATRA (day 1-4) and daratumumab//gG 1 (day 3 )

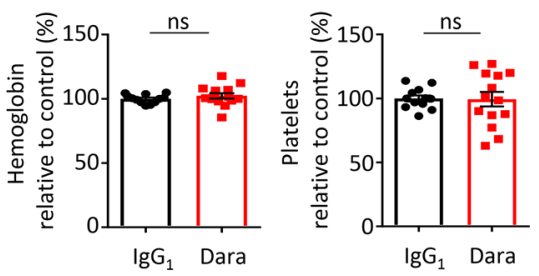

$\mathbf{F}$

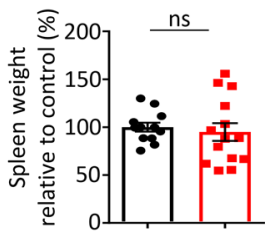

$\operatorname{lgG}_{1} \quad$ Dara

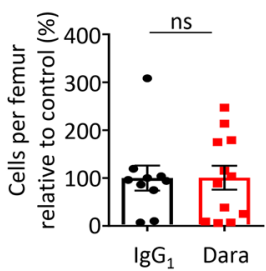

$\lg _{1}$ Dara
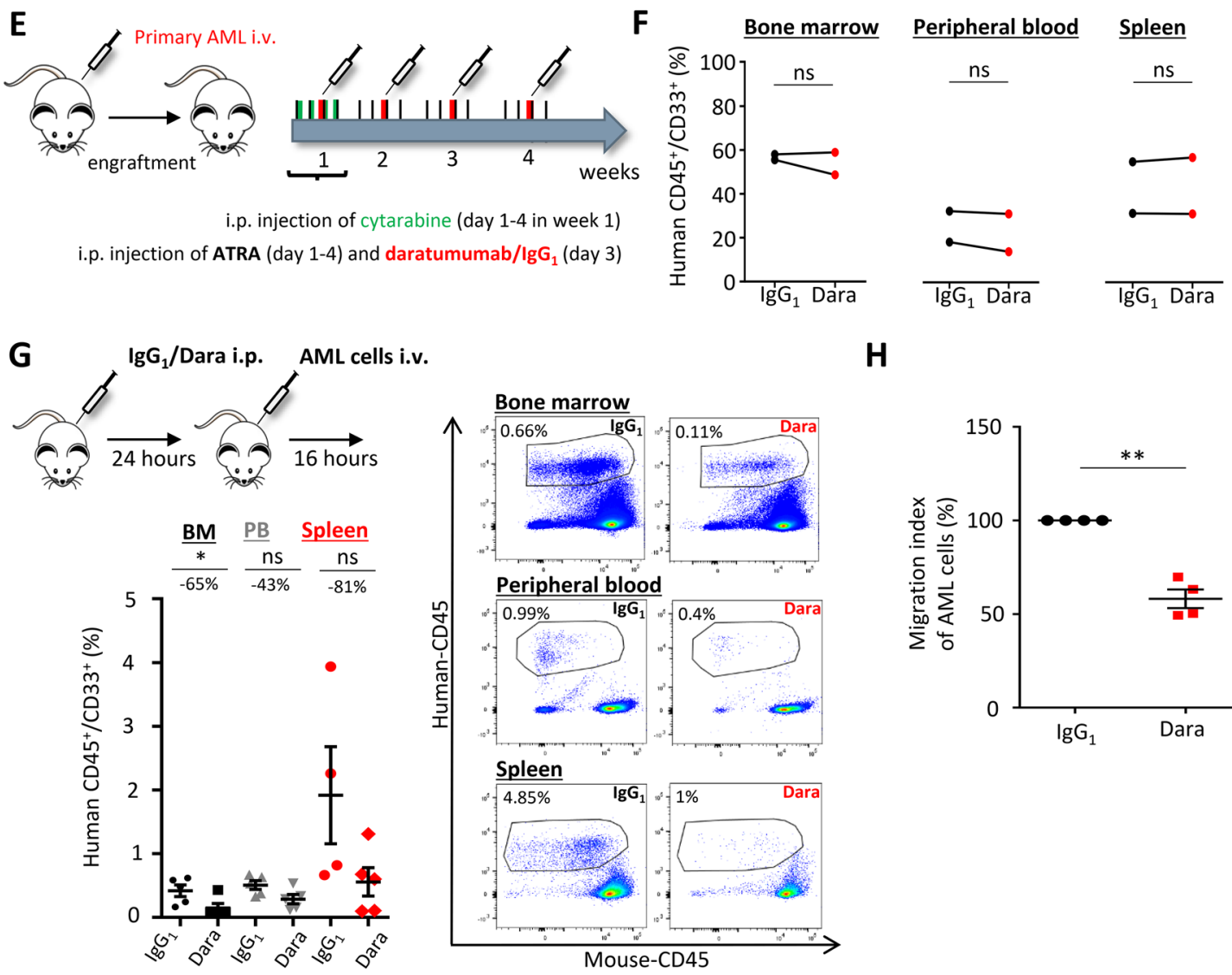

H

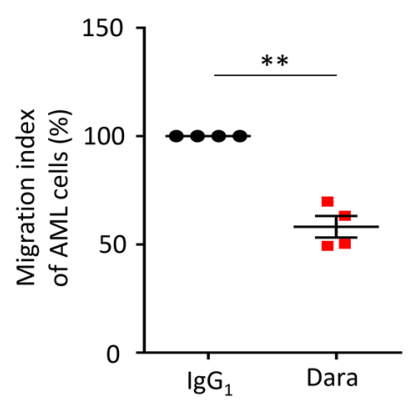


4Figure 4. Daratumumab interferes in AML cell trafficking. (A) Schematic illustration of in vivo treatment protocol with ATRA $(10 \mathrm{mg} / \mathrm{kg})$ for all mice and daratumumab/IgG $(8 \mathrm{mg} / \mathrm{kg})$ after engraftment of primary human AML cells in NSG mice. (B) Representative flow cytometry plots of human hematopoietic engraftment by gating on human $\mathrm{CD}^{+} 5^{+}$cells in bone marrow, peripheral blood and spleen of mice treated with daratumumab or IgG $\mathrm{I}_{1}$ control. (C) Quantification of human AML engraftment $\left(\mathrm{CD} 45^{+} / \mathrm{CD} 33^{+}\right.$cells) in peripheral blood, bone marrow and spleen. Every dot represents mean of all mice per AML sample, $\mathrm{n}=5$ different primary AML samples and $\mathrm{n}=1-5$ mice per sample per group. (D) White blood cells count, hemoglobin levels, platelet count, wet spleen weight and cells per femur of daratumumab or IgG treated mice (data normalized to control, $\mathrm{n}=26)$. (E) Schematic illustration of treatment protocol with cytarabine $(20 \mathrm{mg} /$ $\mathrm{kg})$, ATRA $(10 \mathrm{mg} / \mathrm{kg})$ and daratumumab $/ \operatorname{IgG}_{1}(8 \mathrm{mg} / \mathrm{kg})$ after engraftment of primary human AML cells in NSG mice. (F) Quantification of human AML engraftment $\left(\mathrm{CD} 45^{+} / \mathrm{CD} 33^{+}\right.$cells) in peripheral blood, bone marrow and spleen. Every dot represents mean of all mice per AML sample, $n=2$ different AML samples, 2-3 mice per sample per group. (H) Top, experimental layout to test for interference in AML homing. Below, human AML engraftment $\left(\mathrm{CD} 45^{+} / \mathrm{CD} 33^{+}\right.$cells) in bone marrow (BM), peripheral blood (PB) and spleen $16 \mathrm{~h}$ after transplantation, $\mathrm{n}=5$ mice per group, 2 different AML samples. Right, representative flow cytometry plots of human hematopoietic engraftment by gating on human $\mathrm{CD} 45^{+}$cells in bone marrow, peripheral blood and spleen for each experimental condition. (H) Quantification of transendothelial migration of primary AML cells $(\mathrm{n}=4$, mean of duplicates). Shown is migration index calculated by cells migrated/total cells seeded, normalized to $\operatorname{IgG}_{1}$ control (each dot represents the mean of triplicates). Data are shown as mean \pm SEM. n.s., not significant, ${ }^{*} \mathrm{p}<0.05,{ }^{* *} \mathrm{p}<0.01$ as determined by Wilcoxon signed-rank test $(C)$, paired students t-test $(D, G)$ and MannWhitney-U Test (F, H). See also Supplementary Fig. S6.

we found that daratumumab elicits phagocytosis of AML blasts and seems to interfere with AML circulation. CD38 was shown before to regulate migration of chronic lymphocytic leukemia cells through interaction with its ligand $C D 31^{23}$, which is highly expressed on endothelial cells and which are significantly increased in AML bone marrow ${ }^{24}$. In this regard, it remains unclear how daratumumab interferes with the communication of AML cells with mesenchymal stroma cells, at least in our model, transfer of mitochondria is not significantly affected. Applying daratumumab in vivo in a xenograft transplantation model with primary human AML cells did not lead to robust anti-leukemic effects. In contrast, daratumumab showed significant anti-leukemic efficacy in a subcutaneous scaffold implanted with primary human AML cells ${ }^{25}$ and xenograft transplantation model with AML cell lines with early therapeutic intervention ${ }^{26}$. Based on the hypothesis that daratumumab might exert its highest anti-leukemic efficacy at low disease burden, we combined daratumumab with a chemotherapeutic agent, but were still missing robust anti-leukemic effects. Given the diversified immunomodulatory function of $\mathrm{CD} 38$, it remains unclear to what extent the actual efficacies of daratumumab in AML are recapitulated in immunodeficient NSG mice lacking functional B cells, T cells and natural killer cells. Furthermore, lacking human phagocytizing cells in NSG mice could explain the missing anti-leukemic activity of daratumumab. Therefore, further investigation to find the appropriate model and context to test daratumumab in AML is still required.

\section{Methods}

Patient samples. Primary patient samples were obtained from peripheral blood of newly diagnosed AML patients and non-leukemic donors after informed consent of all subjects according to institutional guidelines. The study was carried out in accordance with relevant guidelines and regulations, with an approved protocol of the University of Duisburg-Essen ethics committee. Mononuclear cells (MNC) were purified using Lymphoprep $^{\text {tw }}$ (STEMCELL Technologies, Vancouver, Canada). Patient characteristics are summarized in Supplemental Table 1.

In vivo treatments. All animal experiments were approved by the responsible local government authorities, namely the Landesamt für Natur, Umwelt und Verbraucherschutz Nordrhein Westfalen. All animal experiments were carried out in accordance with the relevant guidelines and regulations and complied with the ARRIVE guidelines. NOD-scid Il2 $\mathrm{Rg}^{-1-}$ (NSG) mice were bred and used in the animal care facility at the University Hospital Essen. Human mononuclear cells derived from untreated AML patients were depleted from $\mathrm{CD}^{+}$cells using $2 \mu \mathrm{l}$ OKT3/1 $\times 10^{6}$ cells (BioLegend, San Diego, USA) and intravenously transplanted into NSG mice. Bone marrow and spleen cells were harvested from leukemic mice and used for secondary transplantation without prior conditioning. Leukemic mice were equally randomized according to their peripheral blood leukemia burden to each treatment group. Daratumumab $(8 \mathrm{mg} / \mathrm{kg})$ or $\mathrm{IgG}_{1}$ control antibody were given once weekly intraperitoneally for 4 weeks, while all-trans-retinoic acid (ATRA) was administered at $10 \mathrm{mg} / \mathrm{kg}$ intraperitoneally for 4 consecutive days per week for a total of 4 weeks (Fig. $4 \mathrm{a}$ ). In a second experimental approach, cytarabine was added to the first week of treatment, administering $20 \mathrm{mg} / \mathrm{kg}$ intraperitoneally for 4 consecutive days (Fig. 4e). Six days after end of treatment, mice were sacrificed and bones, spleen and peripheral blood were harvested for further processing.

In vivo homing assay. NSG mice were treated with daratumumab or $\operatorname{IgG}_{1}(8 \mathrm{mg} / \mathrm{kg}), 24 \mathrm{~h}$ later mice were transplanted with primary human AML cells. After $16 \mathrm{~h}$, mice were sacrificed and leukemic infiltration in bone marrow, spleen and peripheral blood was assessed by flow cytometry.

Statistical analyses. All data are shown as mean \pm SEM. Shapiro-Wilk test was applied as a test of normality. In case of normality, Student's t test was applied for comparisons between two groups. Mann-Whitney 
$\mathrm{U}$ test and Wilcoxon signed-rank test were applied for nonparametric unpaired and paired analyses. Multiple comparisons were calculated using the one-way ANOVA test. Analyses were performed with GraphPad Prism software (San Diego, USA). ${ }^{*}<0.05,{ }^{* *} \mathrm{p}<0.01,{ }^{* * *} \mathrm{p}<0.001,{ }^{* * *} \mathrm{p}<0.0001$.

\section{Data availability}

The datasets generated during the current study are available from the corresponding author on reasonable request.

Received: 4 May 2021; Accepted: 19 October 2021

Published online: 11 November 2021

\section{References}

1. Dohner, H. et al. Diagnosis and management of AML in adults: 2017 ELN recommendations from an international expert panel. Blood 129, 424-447. https://doi.org/10.1182/blood-2016-08-733196 (2017).

2. Herold, T. et al. Validation and refinement of the revised 2017 European LeukemiaNet genetic risk stratification of acute myeloid leukemia. Leukemia 1, 1. https://doi.org/10.1038/s41375-020-0806-0 (2020).

3. Ishikawa, F. et al. Chemotherapy-resistant human AML stem cells home to and engraft within the bone-marrow endosteal region. Nat. Biotechnol. 25, 1315-1321. https://doi.org/10.1038/nbt1350 (2007).

4. Hanoun, M., Maryanovich, M., Arnal-Estape, A. \& Frenette, P. S. Neural regulation of hematopoiesis, inflammation, and cancer. Neuron 86, 360-373. https://doi.org/10.1016/j.neuron.2015.01.026 (2015).

5. Hanoun, M. et al. Acute myelogenous leukemia-induced sympathetic neuropathy promotes malignancy in an altered hematopoietic stem cell niche. Cell Stem Cell 15, 365-375. https://doi.org/10.1016/j.stem.2014.06.020 (2014).

6. Chen, Y. et al. Acute myeloid leukemia-induced remodeling of the human bone marrow niche predicts clinical outcome. Blood Adv. 4, 5257-5268. https://doi.org/10.1182/bloodadvances.2020001808 (2020).

7. Habbel, J. et al. Inflammation-driven activation of JAK/STAT signaling reversibly accelerates acute myeloid leukemia in vitro. Blood Adv. 4, 3000-3010. https://doi.org/10.1182/bloodadvances.2019001292 (2020).

8. Piedra-Quintero, Z. L., Wilson, Z., Nava, P. \& Guerau-de-Arellano, M. CD38: An immunomodulatory molecule in inflammation and autoimmunity. Front. Immunol. 11, 597959. https://doi.org/10.3389/fimmu.2020.597959 (2020).

9. Malavasi, F. et al. Evolution and function of the ADP ribosyl cyclase/CD38 gene family in physiology and pathology. Physiol. Rev. 88, 841-886. https://doi.org/10.1152/physrev.00035.2007 (2008).

10. Keyhani, A. et al. Increased CD38 expression is associated with favorable prognosis in adult acute leukemia. Leuk Res. 24, 153-159 (2000).

11. Rajkumar, S. V. Multiple myeloma: 2020 update on diagnosis, risk-stratification and management. Am. J. Hematol. 95, 548-567. https://doi.org/10.1002/ajh.25791 (2020).

12. van de Donk, N. \& Usmani, S. Z. CD38 Antibodies in Multiple Myeloma: Mechanisms of Action and Modes of Resistance. Front. Immunol. 9, 2134. https://doi.org/10.3389/fimmu.2018.02134 (2018).

13. Kishimoto, H. et al. Molecular mechanism of human CD38 gene expression by retinoic acid. Identification of retinoic acid response element in the first intron. J. Biol. Chem. 273, 1429-1434 (1998).

14. Fens, M. H. et al. Angiogenic endothelium shows lactadherin-dependent phagocytosis of aged erythrocytes and apoptotic cells. Blood 111, 4542-4550. https://doi.org/10.1182/blood-2007-06-094763 (2008).

15. Moschoi, R. et al. Protective mitochondrial transfer from bone marrow stromal cells to acute myeloid leukemic cells during chemotherapy. Blood 128, 253-264. https://doi.org/10.1182/blood-2015-07-655860 (2016).

16. Marlein, C. R. et al. CD38-driven mitochondrial trafficking promotes bioenergetic plasticity in multiple myeloma. Can. Res. 79, 2285-2297. https://doi.org/10.1158/0008-5472.Can-18-0773 (2019).

17. Deaglio, S. et al. Human CD38 (ADP-ribosyl cyclase) is a counter-receptor of CD31, an Ig superfamily member. J Immunol 160, 395-402 (1998).

18. Henry, C. J. et al. Aging-associated inflammation promotes selection for adaptive oncogenic events in B cell progenitors. J. Clin. Invest. 125, 4666-4680. https://doi.org/10.1172/JCI83024 (2015).

19. Leimkuhler, N. B. \& Schneider, R. K. Inflammatory bone marrow microenvironment. Hematol. Am. Soc. Hematol. Educ. Program 294-302, 2019. https://doi.org/10.1182/hematology.2019000045 (2019).

20. Cai, Z. et al. Inhibition of inflammatory signaling in Tet2 mutant preleukemic cells mitigates stress-induced abnormalities and clonal hematopoiesis. Cell Stem Cell 23, 833-849. https://doi.org/10.1016/j.stem.2018.10.013 (2018).

21. Reynaud, D. et al. IL-6 controls leukemic multipotent progenitor cell fate and contributes to chronic myelogenous leukemia development. Cancer Cell 20, 661-673. https://doi.org/10.1016/j.ccr.2011.10.012 (2011).

22. Buteyn, N. J. et al. Anti-leukemic effects of all-trans retinoic acid in combination with Daratumumab in acute myeloid leukemia. Int Immunol 30, 375-383. https://doi.org/10.1093/intimm/dxy040 (2018).

23. Malavasi, F. et al. CD38 and chronic lymphocytic leukemia: a decade later. Blood 118, 3470-3478. https://doi.org/10.1182/blood2011-06-275610 (2011).

24. Padro, T. et al. Increased angiogenesis in the bone marrow of patients with acute myeloid leukemia. Blood 95, 2637-2644 (2000).

25. Naik, J. et al. CD38 as a therapeutic target for adult acute myeloid leukemia and T-cell acute lymphoblastic leukemia. Haematologica 104, e100-e103. https://doi.org/10.3324/haematol.2018.192757 (2019).

26. Mistry, J. J. et al. Daratumumab inhibits acute myeloid leukaemia metabolic capacity by blocking mitochondrial transfer from mesenchymal stromal cells. Haematologica 106, 589-592. https://doi.org/10.3324/haematol.2019.242974 (2021).

\section{Acknowledgements}

The authors would like to thank the staff at the IMCES imaging facility for their assistance with expert cell sorting and imaging as well as the Institute of Pathology of the University Hospital Essen for H\&E and reticulum stainings. This work was supported by a grant from the Dr. Werner Jackstädt Foundation. M.F. is supported by the $\mathrm{PhD}$ program ELAN, which is promoted by the Else Kröner-Fresenius Foundation.

\section{Author contributions}

M.F. designed and performed experiments, analyzed data, and wrote the manuscript. Y.C., L.A., M.M., E.B., Y.L. performed experiments, analyzed data. H.C.R., U.D. discussed data and provided valuable input on the manuscript. M.H. designed, performed and supervised the study, discussed data, and wrote the manuscript. 


\section{Funding}

Open Access funding enabled and organized by Projekt DEAL.

\section{Competing interests}

H.C.R. received consulting and lecture fees from Abbvie, Astra-Zeneca, Vertex Novartis, GSK and Merck. H.C.R. received research funding from BioNTech, Gilead and Astra-Zeneca. H.C.R. is a co-founder of CDL Therapeutics $\mathrm{GmbH}$. The remaining authors declare no competing interest.

\section{Additional information}

Supplementary Information The online version contains supplementary material available at https://doi.org/ 10.1038/s41598-021-01300-8.

Correspondence and requests for materials should be addressed to M.H.

Reprints and permissions information is available at www.nature.com/reprints.

Publisher's note Springer Nature remains neutral with regard to jurisdictional claims in published maps and institutional affiliations.

Open Access This article is licensed under a Creative Commons Attribution 4.0 International License, which permits use, sharing, adaptation, distribution and reproduction in any medium or
format, as long as you give appropriate credit to the original author(s) and the source, provide a link to the Creative Commons licence, and indicate if changes were made. The images or other third party material in this article are included in the article's Creative Commons licence, unless indicated otherwise in a credit line to the material. If material is not included in the article's Creative Commons licence and your intended use is not permitted by statutory regulation or exceeds the permitted use, you will need to obtain permission directly from the copyright holder. To view a copy of this licence, visit http://creativecommons.org/licenses/by/4.0/.

(C) The Author(s) 2021 